\title{
SISTEMA DE DIODO LASER SINTONIZABLE EN FRECUENCIA PARA APLICACIONES EN LA ESPECTROSCOPIA
}

\author{
TUNABLE FREQUENCY DIODE LASER SYSTEM FOR \\ SPECTROSCOPY APPLICATIONS
}

\author{
Gabriel Altuna ${ }^{1}$, Ciro Carhuancho ${ }^{2}$, Reynaldo Reyes ${ }^{3}$
}

\begin{abstract}
RESUMEN
En este trabajo, describimos un sistema simple y practico para controlar la operación de un diodo láser en una frecuencia específica mediante el uso de una cavidad óptica externa. Nosotros mostramos que para una corriente y temperatura fijas, el láser puede oscilar selectivamente en frecuencias diferentes controlando la longitud de la cavidad externa. Este sistema láser fue utilizado con éxito en un experimento de espectroscopia de absorción saturada en la línea $D_{2}$ del cesio.
\end{abstract}

Palabras clave.- Espectroscopía, Diodo laser, Cesio, Física atómica.

\begin{abstract}
In this work, we describe a practical and simple system to control the operation of a diode laser in a specific frequency using an external optical cavity. We show that fixing the current and temperature, the laser can oscillate selectively at different frequencies by controlling the longitude of the external cavity. This system laser was used with success in an experiment of absorption saturated spectroscopy at the line $D_{2}$ of cesium.
\end{abstract}

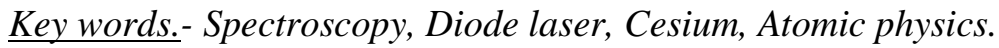

\section{INTRODUCCION}

Mucho de la investigación en física atómica y molecular involucra la interacción de átomos y moléculas con la luz. Fuentes de luz láser que pueden ser sintonizados a una transición atómica particular son ahora una herramienta normal en la mayoría de los laboratorios de física atómica y molecular. Tradicionalmente estas fuentes de luz han sido los láser sintonizables de colorantes (dye laser). Sin embargo, los diodos láser de semiconductores han estado experimentando un crecimiento en eficiencia, potencia y rango de longitudes de onda disponibles, mientras experimentan un decrecimiento en costo. Por otro lado, la reciente mejora en la habilidad para controlar la frecuencia de los diodos láser ha favorecido fuertemente el uso de estas fuentes de luz de bajo costo en varias aplicaciones [1]. En particular, los diodos láser han sido ampliamente empleados en espectroscopia atómica de alta resolución y enfriamiento óptico de átomos neutros [2,3]. Varias técnicas basadas en la realimentación óptica han sido utilizadas para controlar la emisión de estos láseres. Estas técnicas van desde el uso de cavidades tipo Fabry-Perot, redes de difracción, hasta el uso de reflectores simples [1]. Se puede hacer mención también de la técnica de inyección electrónica, en la cual la variación de frecuencia es detectada y corregida por medio de la re-alimentación en la corriente de inyección aplicada al diodo láser [4].

\footnotetext{
${ }^{1}$ Bachiller en Física de la Facultad de Ciencias y Docente de la Facultad de Ingeniería Química y Textil de la Universidad Nacional de Ingeniería, Lima-Perú. ${ }^{2}$ Licenciado en Física de la Facultad de Ciencias y Docente de la Facultad de Ingeniería Química y Textil de la Universidad Nacional de Ingeniería, Lima-Perú. ${ }^{3}$ Doctor en Física por la Pontificia Universidad Católica de Rio de Janeiro, Brasil y Docente de la Facultad de Ingeniería Química y Textil de la Universidad Nacional de Ingeniería, Lima-Perú.
} 
En este caso se utiliza las técnicas de absorción saturada, de reflexión selectiva o de la mezcla de cuatro ondas como referencia para trabar la frecuencia de emisión de los láseres.

Por otro lado, la frecuencia de la radiación emitida por el diodo láser depende tanto de la temperatura como de la corriente aplicada a la unión p-n [1]. Entonces se torna necesaria un control de la temperatura y corriente como un primer paso para obtener una emisión con frecuencia estabilizada.

En este trabajo, describimos el sistema para estabilizar en temperatura y corriente al diodo láser y luego describimos un sistema simple de diodo láser que utiliza una cavidad externa larga (CEL) para sintonizar una frecuencia de emisión que coincida con aquella de una transición atómica. Utilizamos este sistema de diodo láser para realizar un experimento de espectroscopia de absorción saturada en un gas formado por átomos de cesio.

\section{DESARROLLO DEL SISTEMA DE DIODO LASER}

\section{Estabilización en temperatura y corriente}

El primer paso para una buena estabilización de la operación del diodo láser es su montaje mecánico. El diodo láser debe ser fijado en una base de aluminio (o cobre) que servirá de soporte mecánico y también como reservorio térmico. Con la finalidad de obtener el control de temperatura del diodo láser, fue utilizado un circuito que emplea un sensor de temperatura en una red de realimentación que regula la potencia liberada por un elemento refrigerador/calentador tipo Peltier [5]

El funcionamiento del circuito electrónico utilizado para estabilizar la temperatura esta esquematizado en la Fig. 1.

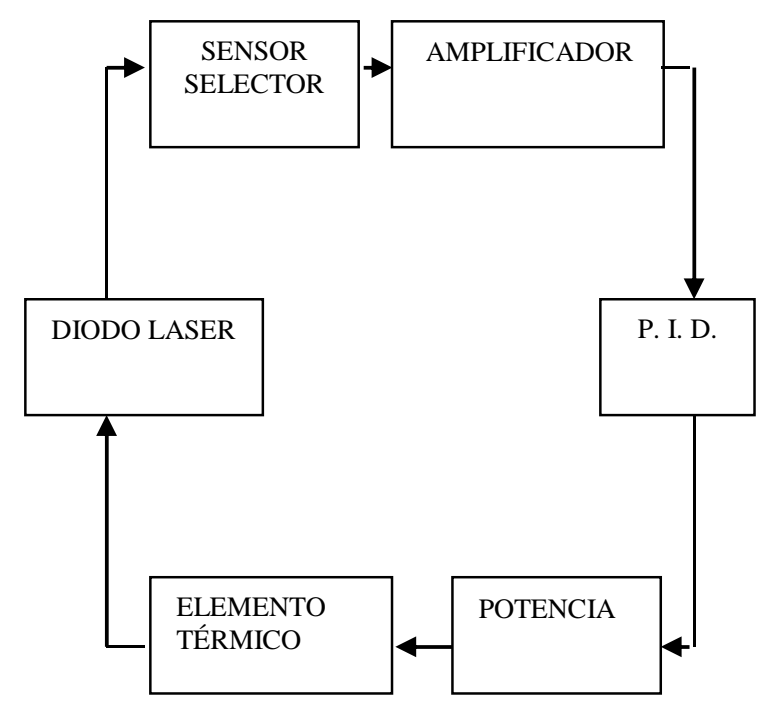

Fig. 1 Diagrama de bloques del circuito eletronico para la estabilização de la temperatura del diodo laser.

La función del Sensor Selector es realizada mediante un circuito con un puente de Wheatstone, como esquematizado en la Fig. 2, donde R1 y R2 son resistencias de precisión (1\%) $\mathrm{y}$ tienen el mismo valor. $\mathrm{R}_{\mathrm{t}}$ es un termistor (NTC), cuya resistencia es función de la temperatura. Este componente tiene la función de sondear la temperatura de la base donde está montado el diodo láser. El selector $\mathrm{R}_{\mathrm{S}}$ es una resistencia variable (un potenciómetro de precisión), con el cual se puede escoger un determinado valor para la resistencia $R_{S} y$, consecuentemente, un valor para la temperatura en que se desea operar.

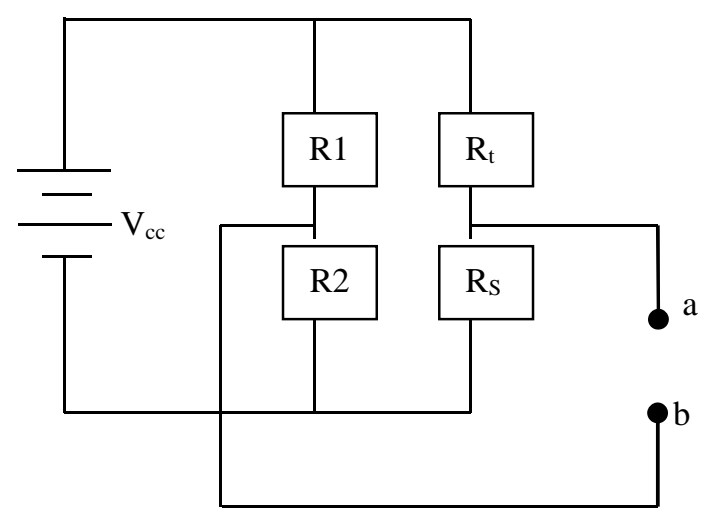

Fig. 2 Esquema del puente de Wheatstone utilizado como sensor/selector. 
En la situación en que los valores de $\mathrm{R}_{\mathrm{S}}$ y $\mathrm{R}_{\mathrm{t}}$ son iguales, el puente esta en equilibrio y la diferencia de potencial (ddp) entre los terminales a y b será nula. Cuando se cambia el valor de $\mathrm{R}_{\mathrm{S}}$, o si cambia la temperatura, lo que implica un variación en $\mathrm{R}_{\mathrm{t}}$, el puente sale del equilibrio $\left(R_{S} \neq R_{t}\right)$; surge entonces una ddp entre los puntos a y b. Esta ddp es la llamada tensión de error $\left(\mathrm{V}_{\mathrm{e}}\right)$ y será mayor cuanto mayor fuera la diferencia entre los valores de $R_{S}$ y $R_{t}$. Por tanto $V_{e}$ indica cual es la diferencia de temperatura entre la base de montaje del diodo láser y la temperatura seleccionada. Así, este circuito funciona como un conversor temperatura/tensión. La tensión de error traduce las fluctuaciones en torno del punto de equilibrio deseado.

El circuito amplificador es responsable por la amplificación de la señal de error, pues el mismo es de orden de algunos milivoltios $\mathrm{y}$, por lo tanto, muy bajo para excitar los circuitos posteriores. Este circuito es constituido simplemente por un amplificador operacional en la configuración de amplificador diferencial, seguido por un "buffer". El diagrama simplificado de este circuito es mostrado en la Fig. 3. La tensión de salida (Ve) tendrá su polaridad en función de la señal de error que es aplicado a los puntos a' y b'. Cuando la tensión aplicada al terminal inversor (punto a') fuera mayor que la tensión aplicada al terminal no-inversor (punto b'), la tensión de salida del primer estado (punto c') será negativa, caso contrario la tensión de salida será positiva. El "buffer" es un operador operacional, instalado en una configuración de amplificador inversor con ganancia unitaria. Este circuito tiene la función de aislar el circuito amplificador del próximo estagio.

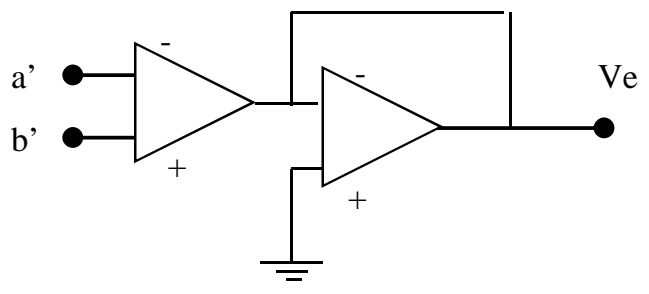

Fig. 3 Circuito simplificado del amplificador.

El circuito proporcional integrador diferenciador (P.I.D.) es responsable por las acciones correctivas en la señal de error amplificado. Para una rápida explicación de sus funciones, utilizaremos el diagrama de bloques representado en la Fig. 4.
El circuito Proporcional es un circuito amplificador cuya salida varia en una relación lineal con la señal de entrada, multiplicada por una constante que es un factor de amplificación, conforme la Ec. (1). Por un ajuste de un potenciómetro $\left(P_{3}\right)$ este factor puede ser alterado.

$$
V_{S}=P_{3} V_{e}
$$

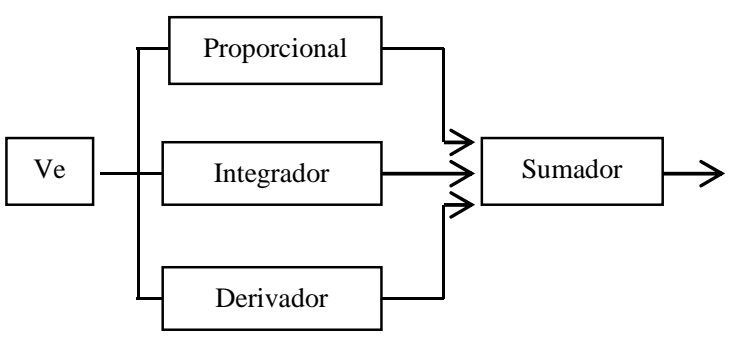

Fig. 4 Diagrama de bloques del circuito PID.

En el circuito Integrador, la salida es la integral a lo largo del tiempo de la señal de entrada, multiplicada por una constante de proporcionalidad, que es la ganancia de integración. Esta ganancia es función de los valores de los condensadores y resistencias asociadas al circuito y puede ser ajustado por un potenciómetro $\left(P_{2}\right)$, conforme la Ec. (2). Este circuito es conocido también como filtro pasa baja.

$$
V_{S}(t)=-\frac{1}{P_{2} C_{2}} \int_{0}^{t} V_{e}(t) d t
$$

El Diferenciador o filtro pasa alta, proporciona una señal de salida que es proporcional a la tasa de variación de la señal presente en su entrada, en este caso, la señal de error. Su respuesta obedece a la Ec. (3):

$$
V_{S}=-P_{4} C_{8} \frac{d V_{e}}{d t}
$$

El Sumador es un circuito que realiza la suma de los tres resultados presentados en las líneas anteriores, teniendo en su salida una señal de corrección del error. Esta señal es transmitida al modulo de potencia. El circuito de Potencia tiene como función proporcionar la corriente necesaria, en función de la señal de error corregido, al 
Elemento Térmico, o sea, al dispositivo responsable por el calentamiento o enfriamiento de la base de montaje del diodo láser.

En el presente montaje, este dispositivo térmico es un elemento "Peltier". El "Peltier" es un dispositivo semiconductor que es capaz de regular el flujo de calor que fluye entre sus dos caras. Este control es función de la corriente y de la tensión aplicada a sus terminales y, de acuerdo con la polaridad de esta tensión, es posible calentar o enfriar una cara en relación a la otra.

Finalmente, en lo que respecta a la estabilización de la temperatura, el montaje mecánico que sirve de soporte al diodo láser también es muy importante para la estabilización de la temperatura. El diodo láser es montado en un pequeño soporte metálico, cuya base debe estar en contacto térmico con el "Peltier".

La otra cara del "Peltier" también precisa ser colocada en contacto térmico con un bloque metálico (aluminio o cobre) que sirve como reservorio de calor. Como la función del "Peltier" es transferir calor de una de sus caras para la otra, este reservorio debe poseer una masa adecuada, lo que es necesario para que exista un buen intercambio de calor.

En lo que se refiere al control de la corriente, se tiene que la estabilización de la corriente aplicada al diodo láser es de suma importancia para tener una buena estabilización de la frecuencia de la radiación emitida [1].

Por lo tanto, las fuentes de corriente utilizadas deben poseer una alta relación señal/ruido y protección contra fluctuaciones bruscas que pueden comprometer el funcionamiento del diodo láser.

El circuito de estabilización de corriente funciona basado en la comparación de una tensión de referencia. Una variación de tensión producida por la corriente de una resistencia sensor, que esta en serie con la carga, es entonces corregida para su nivel de referencia. En la Fig. 5 se muestra la disposición de los sistemas de estabilización de temperatura y corriente conectados al diodo láser.

El circuito de estabilización de temperatura funciona alimentado por una fuente de 12 VDC para los componentes activos del circuito y una fuente de corriente para el "Peltier". La temperatura del diodo láser es monitoreada por medio de un termistor NTC conectado a un multimetro.

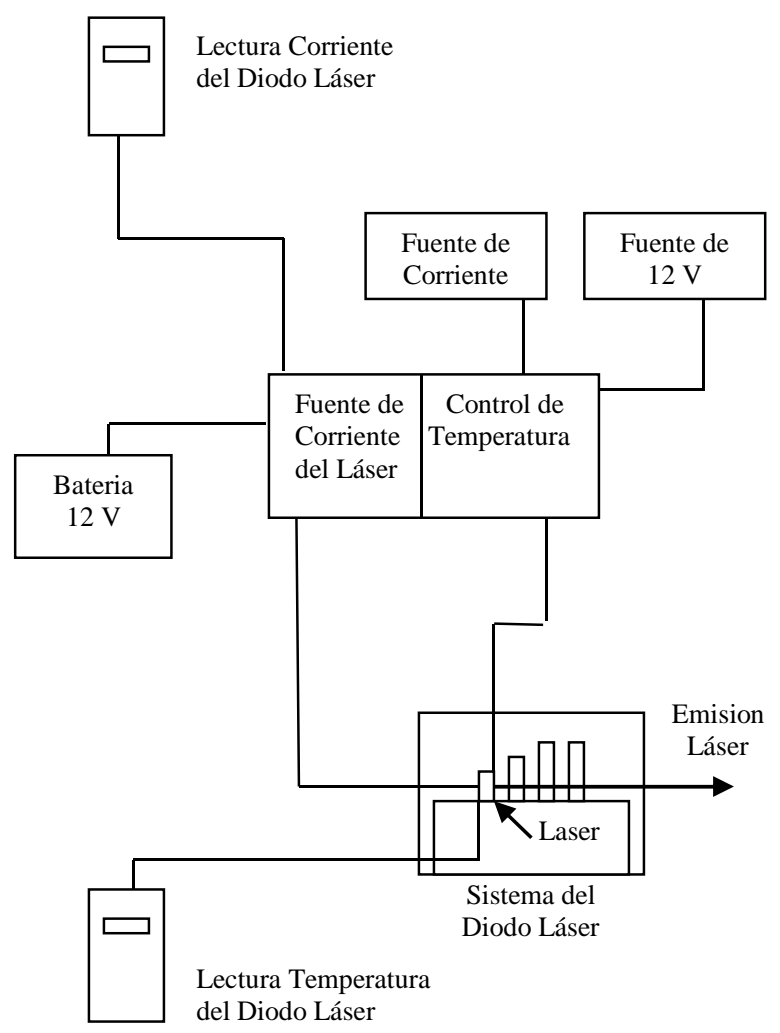

Fig. 5 Disposicion del sistema de diodo laser estabilizado en temperatura y corriente.

La temperatura directa es en Ohmios y con ayuda de un grafico de calibración se obtiene la temperatura en grados centígrados.

El circuito estabilizador de corriente es alimentado por una batería química de $12 \mathrm{~V}$, del tipo utilizado en los automóviles, para una mayor estabilidad de corriente del diodo láser y para eliminar la frecuencia de $60 \mathrm{~Hz}$ de la red eléctrica

\section{Sintonización de la frecuencia por realimentación óptica}

En la Fig. 6 se muestra el sistema de diodo láser que utiliza la técnica de realimentación óptica mediante una cavidad externa larga (CEL) para controlar el ancho de línea y la frecuencia de emisión [1, 6 y 7]. 


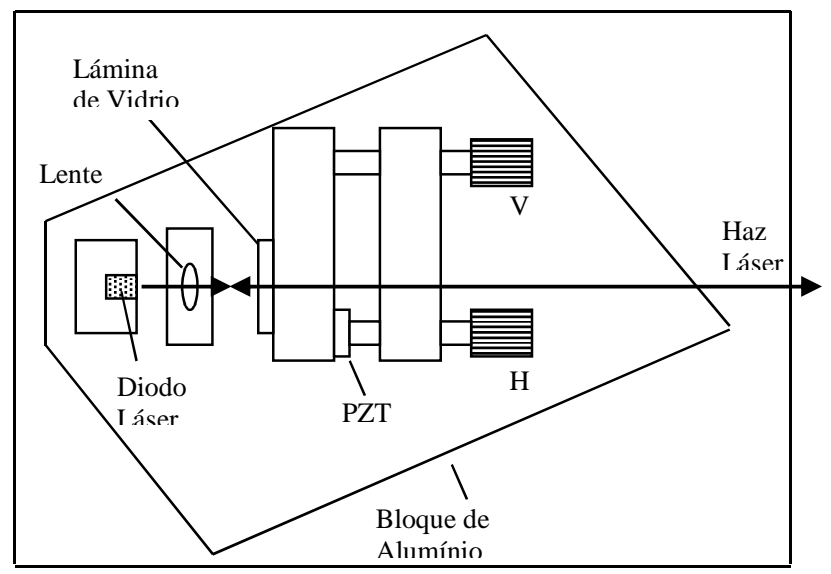

Fig. 6 Sistema de diodo laser con realimentacion optica. $H, V$, tornillos de ajuste horizontal y vertical.

La cavidad externa es formada por una simple lámina de vidrio con faces paralelas, tipo lamina de microscopio, que refleja aproximadamente el 5 $\%$ de la potencia del haz incidente al láser. La lamina de vidrio esta situada a $\sim 5 \mathrm{~cm}$ do diodo láser. El sistema consta de un bloque de aluminio que es utilizado como soporte mecánico y como reservorio térmico. El diodo láser esta montado sobre el dispositivo "Peltier", con el cual se estabiliza la temperatura del diodo. La lamina de vidrio esta montada en un soporte que tiene tornillos micrométricos para los alineamientos vertical y horizontal. Para un ajuste mas fino de la distancia de la cavidad externa fue utilizado un disco transductor piezoeléctrico (PZT). El disco PZT es conectado a una fuente de poder DC para proveer el desplazamiento necesario en la estructura de modos de la cavidad externa, llevando así a la selección de un modo deseado del láser. Para proteger el láser de corrientes de aire y consecuentes variaciones en la temperatura, el sistema esta encerrado dentro de una caja acrílica transparente. El sistema láser $\mathrm{CEL}$ se puede describir como una cavidad láser efectivo con las caras de salida teniendo una reflectividad modulada [6]. Si denotamos las reflectividades de la cara de salida del láser y de la lámina de vidrio por $R_{0}$ y $R$, respectivamente, en el límite de $R_{0}<R$, la reflectividad efectiva de la cara de salida del láser puede ser escrita como [8]:

$$
R_{e f}=R_{0}\left[1+\frac{2\left(1-R_{0}\right)}{\sqrt{R_{0}}} \sqrt{R} \cos \left(\frac{4 \pi L}{\lambda}\right)\right]
$$

donde $L$ es la longitud óptica de la cavidad externa formada por la cara de salida del láser y la lámina, y $\lambda$ es la longitud de onda oscilante.

De la Ec. (4) vemos que las perdidas de la cavidad son periódicamente modulados con un periodo dado por rango espectral libre de la cavidad externa, i.e., $\Delta \lambda_{\mathrm{CE}}=\lambda^{2} / 2 L$. El modo oscilante es elegido haciendo que un modo de la CEL coincida con un modo de la cavidad original del diodo láser; estos modos son separados por $\Delta \lambda_{L}=\lambda^{2} / 2 l$, donde $l$ es la longitud óptica efectiva de la cavidad original del láser. Sin embargo, debido a que para la CEL nosotros tenemos $\Delta \lambda_{\mathrm{CE}}<<\Delta \lambda_{L}$, entonces para tener solo un modo del láser en coincidencia con un modo de la CEL dentro de la curva de ganancia, se debe satisfacer la condición $\Delta \lambda_{L} / \Delta \lambda_{\mathrm{CE}}=L / l . p / q$, donde $p$ y $q$ son enteros.

Bajo esta condición, un cambio en la longitud de la cavidad externa de $\delta L=L \delta \lambda / \lambda=\varepsilon \lambda / 2$, donde el parámetro $\varepsilon(0<\varepsilon<1)$ determina el próximo modo del láser que es mas cercano al otro modo de la $\mathrm{CE}$, hará a la frecuencia del láser saltar a este modo [6].

\section{RESULTADOS EXPERIMENTALES}

El sistema de diodo láser descrito anteriormente es aplicado a un experimento de espectroscopia de absorción saturada [2], con un diodo láser modelo SDL-5401-G1, emitiendo con una longitud de onda nominal en torno de $853 \mathrm{~nm}$. La disposición utilizada para realizar el experimento es descrito en las referencias [2,7,9]; el haz del láser es dividido en dos haces, uno de los cuales es usado como un relativamente intenso haz de bombeo y el otro es usado como un haz débil de prueba. El haz de bombeo es colimado y aplicado a la celda de Cs con un diámetro relativamente grande para saturar la absorción en una región grande de la celda.

El haz de prueba con un pequeño diámetro es aplicado a la celda desde el lado opuesto al haz de bombeo. Cuando la frecuencia del láser es barrido en torno de la línea $\mathrm{D}_{2}$ del $\mathrm{Cs}$ mediante la variación de la longitud de la cavidad óptica externa con el PZT, espectros angostos libres del efecto Doppler pueden ser observados sobre un fondo ensanchado por efecto Doppler en la señal de absorción del haz de prueba (Fig. 7). 


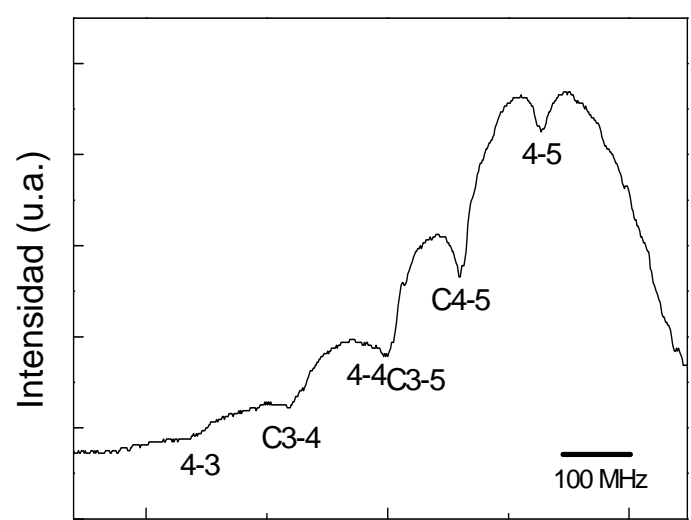

Frecuencia

Fig. 7 Espectro de absorción saturada de la línea $\mathrm{D}_{2}$ del cesio, obtenido utilizando el sistema de diodo láser CEL.

La celda conteniendo los átomos del cesio 133 estaba a una temperatura de $20^{\circ} \mathrm{C}$, siendo así su densidad de $\sim 2 \times 10^{10}$ átomos $/ \mathrm{cm}^{3}$. El haz del láser fue sintonizado usando la CEL en una frecuencia próxima a la línea $\mathrm{D} 2$ del cesio $(\lambda=852.1 \mathrm{~nm})$, con la transición de resonancia $6 \mathrm{~S}_{1 / 2} \rightarrow 6 \mathrm{P}_{3 / 2}$.

El estado fundamental $6 \mathrm{~S}_{1 / 2}$ consiste de estados hiperfinos con $F=3$ y 4 ; el estado $6 \mathrm{P}_{3 / 2}$ consiste de cuatro estados hiperfinos con $F^{\prime}=2,3,4$ y 5 . El ancho de línea natural es $\sim 5.3 \mathrm{MHz}$ y el ancho Doppler es $\sim 400 \mathrm{MHz}$ en $20^{\circ} \mathrm{C}$. La separación hiperfina de $9.1 \mathrm{GHz}$ en el estado fundamental es mucho mayor que el ancho Doppler de la línea de absorción, así que las líneas de absorción de los estados hiperfinos con $F=3$ y 4 en el estado fundamental son bien separadas.

En la Fig. 7 se muestra el espectro de absorción saturada obtenido utilizando el sistema de diodo láser CEL, considerando las transiciones a partir del estado hiperfino fundamental $F=4$. Cuando la frecuencia del láser es barrida sobre la línea de absorción del estado hiperfino fundamental $F=4$, seis resonancias libres del efecto Doppler aparecen sobre un fondo de una señal de absorción con ancho Doppler: tres depresiones (dips) de Lamb [2,9] correspondientes a las transiciones $F=4 \rightarrow F^{\prime}=3,4$ y 5 , y tres resonancias de cruzamiento (crossover resonances) C3-4, C3-5, C4-5 [2,7]. Estos resultados están en concordancia con lo reportado en la literatura [9 y 10], lo cual indica que el sistema de diodo láser descrito presenta un buen desempeño.

\section{CONCLUSIONES}

En este trabajo, hemos reportado sobre la sintonización y la estabilización de la frecuencia de oscilación de un diodo láser. Se ha descrito la estabilización en temperatura y corriente del sistema, lo que permitía luego la sintonización de frecuencia a la línea $\mathrm{D}_{2}$ del cesio mediante el uso de una cavidad externa larga (CEL). Para demostrar el funcionamiento efectivo de este sistema de diodo láser, fue realizado un experimento de espectroscopia de absorción saturada, con resultados similares a lo reportado en la literatura. Este hecho muestra la factibilidad de construir un sistema óptico pequeño, estabilizado en corriente y temperatura, sintonizable en frecuencia, con un costo relativamente bajo. Este sistema de diodo láser puede ser útil para aplicaciones en mediciones de precisión de longitud y velocidad, comunicaciones ópticas, espectroscopia láser, fuente de luz para el bombeo hiperfino en un reloj atómico, espectroscopia de reflexión selectiva [7], enfriamiento de átomos por métodos ópticos, entre otras aplicaciones $[1,11]$.

\section{AGRADECIMIENTOS}

Los autores agradecen a los Profesores Marcos Oriá y Martine Chevrollier del Laboratorio de Física Atómica y Láser, Universidade Federal da Paraíba, Brasil, por el apoyo y colaboración. También agradecen al IGI-UNI. Uno de los autores (R.R.) agradece el apoyo brindado por el Consejo Nacional de Pesquisas (CNPq) de Brasil.

\section{REFERENCIAS}

1. C. Weiman, L. Hollberg, "Using diode lasers for atomic physics", Rev. Sci. Instrum. 62, 1-20 (1991).

2. W. Demtroder, "Laser spectroscopy", Springer (1996).

3. D. Sesko, C.G. Fan, C. E. Weiman, "Production of a cold atomic vapor using diode-laser cooling", J. Opt. Soc. Am. B 5, 1225-1227 (1988).

4. Y. Shevy, H. Deng, "Diode laser with injection current", Optics Letter 23, 472-475 (1998).

5. H. N. de Freitas, "Espectroscopia por mistura de quatro ondas em vapor de 
rubidio", Tesis de Maestria, Universidade Federal da Paraiba, Brasil (1996).

6. L. Viana, S.S. Vianna, M. Oria, J.W.R. Tabosa, "Diode laser mode selection using a long external cavity", Appl. Optics 35, 368371 (1996).

7. R. Reyes, "Espectroscopia de reflexao seletiva em uma interface dieletrico-vapor atomico de cesio", Tesis de Maestria, Universidade Federal da Paraiba, Brasil (1999).

8. A. Olsson, C.L. Tang, "Coherent optical interference effects in external-cavity semiconductor lasers", IEEE J. Quantum Electron. QE-17, 1320-1323 (1981).

9. H. Hori, Y. Kitayama, M. Kitano, T. Yabuzaki, T. Ogawa, "Frequency stabilization of GaAlAs laser using a
Doppler-free spectrum of the Cs- $\mathrm{D}_{2}$ line", IEEE J. Quantum Electron. QE-19, 169-174 (1983).

10. A.M. Akulshin, V.L. Velichanski, A.S. Zibrov, V.V. Nikitin, V.A. Sautenkov, G.G. Kharisov, "Stabilization of the frequency of a highly coherent injection laser using intraDoppler saturated absorption resonances", Sov. J. Quantum Electron. 18, 1214-1215 (1988).

11. R. Reyes, "Fundamentos de la interacción átomo-luz de laser", ISBN 978-9972-33-7727 (1999).

Correspondencia: limaperu773@gmail.com

Recepción de originales: setiembre 2014

Aceptación de originales: diciembre 2014 